\title{
Thermographic investigation of surface temperature of the evaporating liquid layer under the action of gas flow
}

\author{
Aleksei $\mathrm{Kreta}^{1,2^{*}}$, and Yuriy Lyulin ${ }^{2,3}$ \\ ${ }^{1}$ Novosibirsk State University, 630090 Novosibirsk, Russia \\ ${ }^{2}$ Kutateladze Institute of Thermophysics, 630090 Novosibirsk, Russia \\ ${ }^{3}$ National Research Tomsk Polytechnic University, 634050 Tomsk, Russia
}

\begin{abstract}
An experimental study of the temperature field on the surface of horizontal liquid layer (Ethanol) evaporating into gas flow (Air) has been performed. Temperature gradient of the gas-liquid interface has been measured with the help of Titanium 570M IR camera. Shear stresses on gas-liquid interface induced by thermocapillary effect and inert gas flow have been defined.
\end{abstract}

\section{Introduction}

Study of heat and mass transfer through the gas-liquid interface is one of the most important problems in the present time. The intensive evaporation from the liquid surface into the gas flow induces various convective flows within the liquid. Two mechanisms have been spotted to be responsible for the convection. One of them is the gravity that acts essentially on density differences due to temperature gradients, the other one is the thermocapillarity originated by the temperature dependence of the surface tension at the liquid interface. The convective motions caused by buoyancy are known as the Rayleigh-Bénard convection. When the convection is induced by the surface tension, it is referred to as Marangoni-Bénard convection. In the most of investigations the fluid layers are steadily heated from below with a static temperature gradient and without evaporation. The evaporating fluid layers with nonlinear temperature gradient have much less been studied due to the complexity of the problem. The works [1] report the experimental results concerning the effects of evaporation on the thermocapillary convection in a thin liquid layer. It was found that evaporation is not only a means of cooling the layer surface, but also a driving power for the development of the convective instabilities.

The problem of convection of the evaporating liquid layer under the action of the inert gas flow is much more complicated than described above, because includes also the shearstress effect induced by gas flow [2]. The coupling between evaporation and convection cased by a shear-stress, thermocapillary and buoyancy effects in the liquid layer, has a direct effect on the evaporation flow rate, being relevant, for example, for heat-pipe grooves and thin-film evaporators. The increased interest to the problem is caused by

*Corresponding author: alexy.slav@yandex.ru 
experiments under microgravity conditions [3-5], some experiments under normal gravity and the preparation of experiments on the International Space Station in the framework of the scientific project "Convection and Interfacial Mass Exchange" (CIMEX) of the European Space Agency [6]. These experiments are aimed to investigate the features of convective flows of a fluid in an open cavity and in an open horizontal layer under conditions of a co-current gas flow and evaporation. The processes of convection, accompanied by evaporation at the interface, are actively studied experimentally [7], numerically $[8,9]$ and theoretically in the present time $[9,10]$.

The aim of this work is to experimentally study the structure of convective flows and to measure temperature distribution on the surface of horizontal liquid layer under the action of the gas flow using IR camera.

\section{Experimental setup}

The experimental investigations were conducted on the setup shown in Fig. 1. The setup consists of the following components: test section, gas and liquid loops, data acquisition system, thermal stabilization system, and optical techniques.

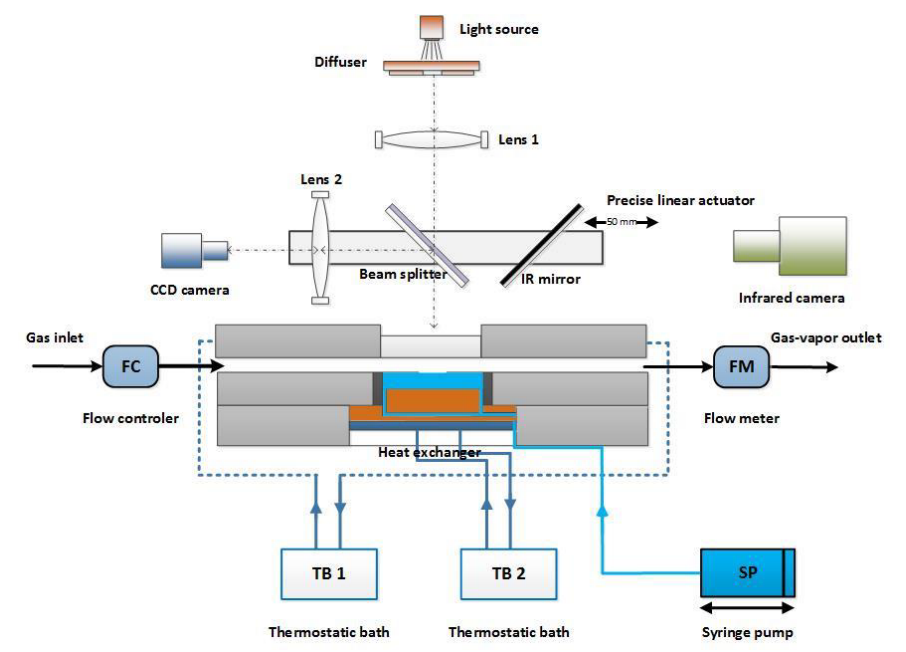

Fig. 1. Scheme of the experimental setup.

Pure gas enters to the test section from the compressor. The mass flow controller sets flow rate of the gas at the inlet of the test section. The liquid is injected into the test section by a precision syringe pump. The working liquid evaporates under the gas flow and the vapor-gas mixture flows to the outlet of the gas channel in test section. The vapor-gas mixture flow at the outlet is measured by a mass flow meter. The liquid temperature in the test section is determined by the temperature of the substrate base, which controlled with the help of thermoelectric module. The position of the interface level is controlled with an accuracy of 10 micrometres using a Schlieren technique and the syringe pump [7].

Measurements of the temperature distribution on gas-liquid interface were conducted with the help with of Titanium 570M IR camera. An infrared wavelength region is 3-5 micrometres. Infrared imaging of liquid surface was performing through quartz optical window that implemented in the top cover of the test cell. IR camera was placed on the optical tabletop horizontally and measuring of the surface temperature of the liquid was contacted with the help of the IR mirror. The IR mirror was inclined to 450 to infrared camera and liquid surface. Optical components of the Schlieren technique and IR mirror were mounted on an optical platform of the linear actuator. Linear actuator is controlled 
from personal computer and by special software. The optical platform moved in the range of $50 \mathrm{~mm}$ and speed of $104 \mathrm{~mm} / \mathrm{s}$ in horizontal direction. Initially, the platform was set in position where optical axes of Schlieren technique coincide and the interface visualized. When a flatness of interface and the stationary regime of the experiment were established the linear actuator moved the platform in position where optical axes of the IR camera and mirror also coincide and measurement of surface temperature was running.

\section{Results and discussion}

The experiments were conducted at atmospheric pressure in a test section, and a $5 \mathrm{~mm}$ thick liquid layer. Ethanol liquid was used as the working liquid. The evaporation surface area was $100 \mathrm{~mm}^{2}$ with the corresponding $10 \times 10 \mathrm{~mm}$ cut-out in the plate. The gas flow rate was in the range $100-1000 \mathrm{ml} / \mathrm{min}$ that corresponds to gas average velocities for 0.0138 to $0.138 \mathrm{~m} / \mathrm{s}(\mathrm{Re}=2.8-28)$, respectively. The temperature of the "liquid-gas" system has changed from $20^{\circ} \mathrm{C}$ to $40^{\circ} \mathrm{C}$. The difference between the temperature of the gas and liquid does not exceed $0.1^{\circ} \mathrm{C}$.

Fig. 2 shows a thermogram of the liquid surface at the intensive evaporation. The IR image snapshot does not explain all dynamic of the process. Analyzing of the video the movement of gas-liquid interface in counter-current direction to the gas flow is observed.

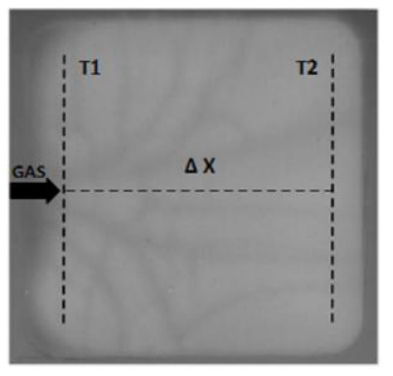

Fig. 2. The thermogram of the evaporating liquid layer.

In Fig. 3 a typical temperature distributions of the interface in the direction of gas flow are plotted for the various temperatures. It is shown the differences in the interfacial temperature. Intensive evaporation induces temperature decreasing in initial of the evaporation surface and temperature gradient is appeared. In this case the thermocapillary effect on the gas-liquid interface takes place.

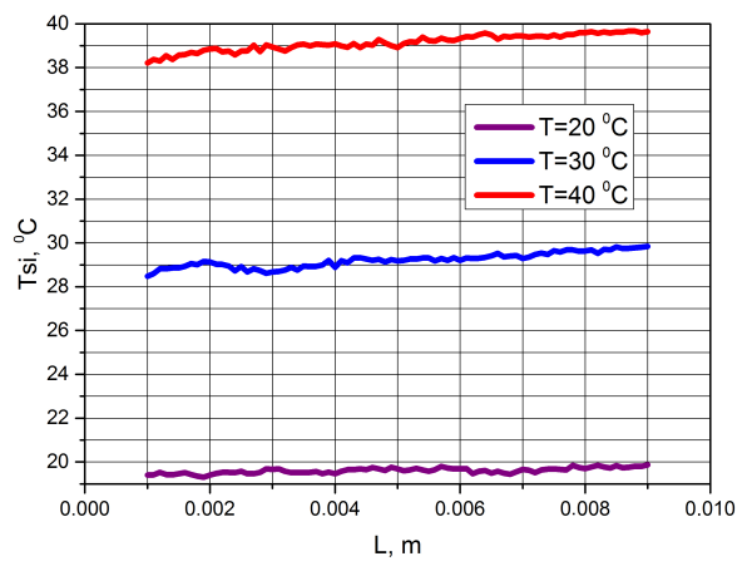

Fig. 3. The temperature distribution on the surface of the evaporating liquid layer. 
The shear stress on the liquid surface $\tau_{\text {sur }}$ caused by the thermocapillary effect can be estimated as:

$$
\tau_{\text {sur }}=\frac{\delta \sigma}{\delta T} \frac{\delta T}{\delta x}
$$

where, $\sigma_{T}=\frac{\delta \sigma}{\delta T}$ - temperature coefficient of surface tension is equal $1,14 \times 10^{-4} \mathrm{~N} / \mathrm{mK}$.

The temperature gradient is defined as: $\frac{\delta T}{\delta x}=\frac{T_{2}-T_{1}}{\Delta x}$. Points of the temperature measuring and length $x=0,008 \mathrm{~m}$ are shown in Fig. 2 .

Shear stresses on the liquid surface induced by gas flow can be estimated as [11]:

$$
\tau_{g}=\eta \frac{d V}{d y}=-\frac{H}{2} \frac{\delta p}{\delta x}
$$

where, $H=0,005 \mathrm{~m}$ is height of gas channel; $\eta$-dynamic viscosity.

Pressure gradient is calculated from the formula: $V_{g}=-\frac{H^{2}}{12 \eta} \frac{\delta P}{\delta x}$, where average gas velocity $V_{g}, \mathrm{~m} / \mathrm{c}$ is known value. It is assumed that in the gas channel the Poiseuille flow is realised. The gas-liquid interface is considered as a rigid surface.

Fig. 4 shows the relationship of forces shear stresses associated with the temperature gradient at the surface of the liquid layer to the forces of shear stresses caused by friction on the surface of the gas flow at different temperatures system "liquid - gas" and at different Reynolds numbers.

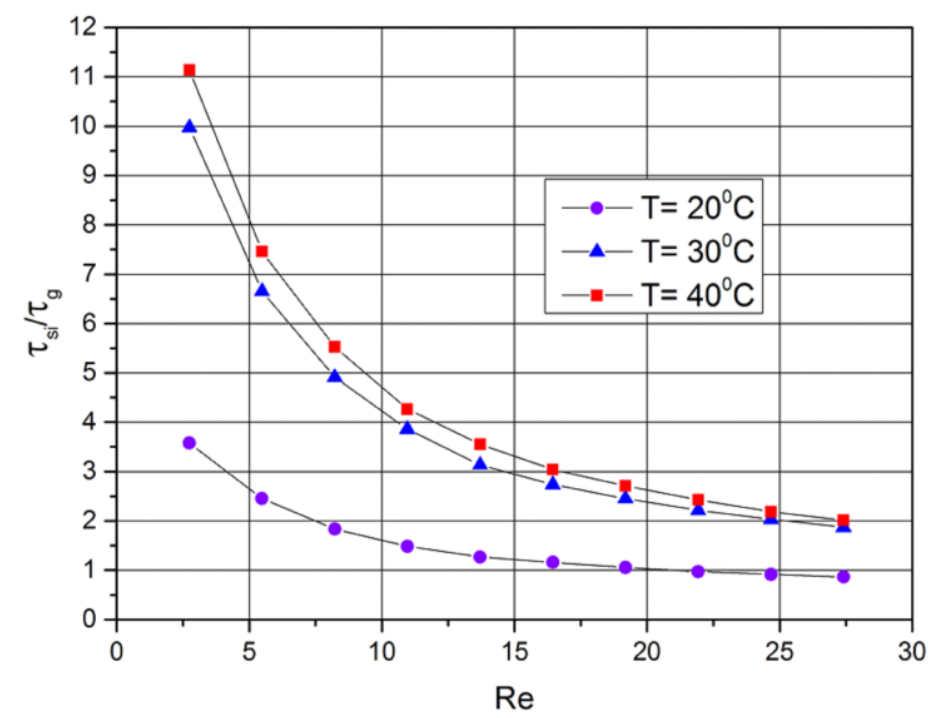

Fig. 4. Shear stresses.

The plot show that with the increase of the gas flow relationship the thermo-capillary shear stress to the gas shear stress are decreased. It can be seen that when the small gas 
Reynolds the thermocapillary shear stresses are bigger than gas shear stresses in several times. This means that thermocapillary forces dominate at low gas velocities. When the maximum gas flow rate relationship the thermo-capillary shear stress to the gas shear stress are significantly decreased. However, thermocapillary forces all the same give a decisive contribution to the development of convection currents in the liquid layer.

\section{Conclusions}

Experiments were carried out to study the influence of the gas flow velocity and temperature system liquid-gas on the temperature distribution on the interface. Infrared study and measurements of the temperature field on the surface of horizontal liquid layer evaporating into gas flow has been performed. Temperature gradient of the gas-liquid interface has been measured with the help of Titanium 570M IR camera. Shear stresses on gas-liquid interface induced by thermocapillary effect and inert gas flow have been defined. It is shown that the main factor influencing on the development of convection in the liquid layer under the action of gas flow is thermocapillary effect.

The work was financially supported by the Russian Ministry of Education and Science (Project identifier RFMEFI61314X0011).

\section{References}

1. N. Zhang, D.F. Chao, Int. Commun. Heat and Mass 26, 1069 (1999)

2. O.A. Kabov, Int. J. Emerging Multidiscip. Fluid Sci. 2,161 (2010)

3. L. Joannes, O. Dupont, S. Gorza, P. Colinet, J. Legros, T. Lanen, H. Oksuzoglu, Proceedings of Fringe 2001, 4th international workshop on automatic processing of fringe patterns, 506 (2002)

4. K. Löth, B. Larsson, H. Schneider, O. Janson, Y. Houltz, P. Colinet, C. Iorio, L. Joannes, O. Dupont, Proceedings of the 53rd international astronautical congress, Houston, Texas (The World Space Congress, (2002)

5. P. Colinet, L. Joannes, C.S. Iorio, B. Haut, M. Bestehorn, G. Lebon, J.C. Legros, Adv. Space Res. 32, 119, (2003)

6. T. Balázs, Microgravity Sci. Technol. 24, 189 (2012)

7. Yu.V. Lyulin, O.A. Kabov, Int. J. Heat Mass Tran. 70, 599 (2014).

8. H. Machrafi, C.S. Iorio, P.C. Dauby, Interfacial Phenomena and Heat Transfer 2, 1 (2014)

9. O.N. Goncharova, O.A. Kabov, Int. J. Heat Mass Tran. 53, 2795 (2010)

10. O.N. Goncharova, M. Hennenberg, E.V. Rezanova, O.A. Kabov, Interfacial phenomena and heat transfer 1, 317 (2013)

11. L. Landau, E. Lifshitz, Hydrodynamics (Science, Moscow, 1986) 